\title{
Ageing Societies and Technological Innovation
}

\section{Concepts of Ageing Society and Old Age}

Demographic change has been declared as one of the main challenges for Western societies by politicians, journalists, industry and academia alike (Harper, 2006). The fact that societies are ageing is demonstrated through the growing number of older citizens relative to the rest of society. For example in Europe, the percentage of citizens aged 65 and above is projected to rise from $16 \%$ in 2010 to $29.3 \%$ in 2060 and the percentage of citizens aged over 80 is projected to increase from $4.1 \%$ in 2010 to $11.5 \%$ by 2060 (Creighton, 2014).

Most stakeholders agree that this change entails consequences for our health care and social systems as well as "labour supply, family and household structures, health and welfare service demand, patterns of saving and consumption, provision of housing and transport, leisure and community behavior, networks and social interaction" (Harper, 2006, p. 1). Associated fears of the financial burden to social security and health care systems are prominent. At the center of the discourse are "calls for action on the "ageing society" (Moreira, 2017, p. 2) to tackle the "demographic burden" (Harper, 2006, p. 19). However, scholars in critical and social gerontology argue that most of the alarmist rhetoric about demographic ageing and projected social implications are based on flawed assumptions about older people (e.g. their ability to contribute to their communities) and the ageing process (e.g. as solely described in terms of decline and long-term care needs) (Harper, 2006; Moreira, 2017). For example, Harper (2006) argues that the provision of health care for older people is only one aspect of demographic change, as most people will live the remainders of their live after retirement in "reasonable health, with limited disability". She proposes to shift focus, e.g. to inflexible retirement systems which cause a rising dependencies of older people rather than their unwillingness or incapacity to work. 
Who is considered old, differs substantially across countries, depending for example on the median age and life expectancy. Neugarten defined in 1974 a distinction between the "young-old" versus the "old-old" (Neugarten, 1974), which was later adopted by Laslett in his distinction between the third and the fourth age (Laslett, 1987, 1991). Persons in their third age, usually starting with retirement, are still relatively healthy and have time to follow their hobbies, social activities and for life-long learning. In contrast, persons in their fourth age are characterised by declining mental and physical health, ending in final dependence, decrepitude, and death. Hence, there is a

principal binary opposition in the way later life is represented; namely the distinction between a fit, healthy and productive later life and an old age dogged by ill health, incapacity and neediness (Higgs \& Gilleard, 2015, p. 10).

Whereas Laslett considered the third age a distinct life stage, he did not see it bounded by chronological age. Rather the transition from third to fourth age is a fluid and contingent process only depending on a persons' abilities. Others have operationalised the idea and calculate the third-fourth age division by "the chronological age at which $50 \%$ of the birth cohort are no longer alive" (Baltes \& Smith, 2003, p. 125 as cited in Higgs and Gilleard (2015)). Higgs and Gilleard (2015) argue that both concepts can be understood as social imaginaries about what it means to grow old. In the third age, social agency plays a critical role, it is very much influenced by lifestyle choice and identity:

Third age narratives and practices often sustain different identities and lifestyles and draw upon points of reference unrelated to agedness - such as one's sexuality, gender or ethnicity, or the uses of one's cultural and social capital - many implicitly reference ageing and old age (Higgs \& Gilleard, 2015, p. 117).

There are a number of dividing practices between the third and fourth age which are reiterated in public discourse but also individual identity building. Most notable is the discourse around "successful ageing", often also called "active ageing" which ascribes in an almost normative way a particular set of practices to performing third age identities. The distinction has received some criticism: On the hand it is argued that it destroys the "bond" between old people and their "shared experience of discrimination and social and cultural marginality" (Higgs \& Gilleard, 2015, p. 11). Others have pointed out that the distinction between third and fourth age, conceals other, more fundamental differences such as socio-economic divisions in society (e.g. financial capital, social capital etc.). Holstein, Parks, and Waymack (2011) argue that

the paths to old age are deeply shaped by social location with some locations providing the foundation for a 'successful' old age while others make it very difficult especially for lower income women and people of color (p.xv).

In contrast, Higgs and Gilleard (2015) argue that much of the distinction between third and fourth age "lies outside these classical sociological structures" as for example "the cultures of the third age cannot be easily read off from an infrastructure of class, cohort and community" (Higgs \& Gilleard, 2015, p. 115). 
Increasingly the image of the frail, lonely and dependent elderly is accompanied by the notion of an active, healthy and capable older person. This has had a strong influence on how people perceive of themselves and their ageing bodies.

How we experience our aging bodies is thus complex, influenced by structural, institutional, and cultural forces and the myriad interactions that occur in the overlapping and discrete contexts in which we live. Our embodied selves shape and are shaped by these forces and interactions (Holstein et al., 2011, p. xiv).

The process of ageing is hence not only biological but also a symbolic, discursive and cultural phenomenon (Höppner \& Urban, 2018; Wanka \& Gallistl, 2018). Certain expectations and bodily norms — such as ageing actively and healthy - provide the basis for how age is being performed as social practice, through interactions with other people (Schroeter, 2005; Schroeter \& Künemund, 2010). In this understanding old age is conceptualised as a social imaginary, which is a way

people imagine their social existence, how they fit together with others, how things go on between them and their fellows, the expectations that are normally met, and the deeper normative notions and images that underlie these expectations (Taylor, 2003, p. 23).

What is receiving increasing scholarly attention is the materiality of age and ageing in the context of everyday life (e.g. Höppner \& Urban, 2018). It moves away from social constructivist approaches to extent the frame of analysis beyond discursive practices, to ageing bodies and material environments: Within material gerontology things, technology and spaces are considered as part of sociomaterial assemblages. Ageing is understood as a "material-discursive practice" (Barad, 2003, 2007); it does not exclusively happen in a human body, but rather

[...] age and ageing are co-products of human interactions, discourses, things, technical artifacts, possessions, and mobilities, among other things. From such a perspective, ageing becomes a complex process in which human bodies and all kinds of materiality can be involved (Höppner \& Urban, 2018, p. 2).

For the purpose of this book age has "analytical value" as it identifies "birth-cohort membership" and the potential of shared life-experiences such as the introduction of particular technologies during specific life events like childhood, youth or work life (Harper, 2006, p. 3). Bolin (2017) proposes the term "media generation" and argues that people of the same age group share media experiences throughout their life-course: "media technologies and content have become increasingly important components in everyday life, and hence also in the process of generation formation" (p. 5). Since age and ageing are co-products of human interactions, shared experiences, discourses and technologies, a "generational identity" is formed and shapes certain habits and modes of media use. Similarly, Hepp, Berg, and Roitsch (2017) argue that the concept of media generation is useful to describe groups of people who share specific experiences based on their personal "media biographies" and hence "developed a shared self-image as a media generation" (p. 109). 


\section{Ageing and Information Technologies}

Overall are controversies about the "ageing society" not only a sign of its importance for our societies, but also shape "the way in which we approach, understand and manage ageing processes in society" (Moreira, 2017, p. 5). Moreira recommends to study the knowledge making practices, tools, technologies and knowledge making institutions that co-construct different positions about the "ageing society". Similarly others have argued that in and of itself ageing is not a problem for which a solution is required, but for example, technology-driven discourses frame ageing in this particular way (Höppner \& Urban, 2018; Neven \& Peine, 2017; Neves \& Vetere, 2019; Wanka \& Gallistl, 2018). For example, older adults have become a key target group for technology design in the area of medical and assistive technologies (Bischof, 2017; Cozza, De Angeli, \& Tonolli, 2017). Not surprisingly have many sought to develop technological solutions as response to the perceived challenges and opportunities of an ageing population (e.g. robot companions to address loneliness as discussed by Turkle, 2011). Most of these technological solutions frame ageing "as a 'problem' that can be managed by technology" (Vines, Pritchard, Wright, Olivier, \& Brittain, 2015, p. 2). However, the relation between ageing and technology has been conceptualised in very different ways.

\section{Technology Acceptance as Reasoned Action}

The rational choice paradigm is the most commonly used paradigm in social gerontology (Wanka \& Gallistl, 2018). It explains the differences in the uptake of technologies as a matter of individual choice, determined by the subjectively perceived usefulness and the subjectively perceived ease of use/level of difficulty of any given technology (e.g. Davis \& Venkatesh, 1996; Wang, Rau, \& Salvendy, 2011). In particular, the aspect of "ease of use" has been picked up by a number of technology companies, developing user-friendly digital media technologies (Peine, Rollwagen, $\&$ Neven, 2014) and aiming to overcome people's conviction that certain types of technology are "obviously not for me" (Neven, 2010).

This paradigm however, underestimates the influence of social structures on active ageing and technology use as it solely focuses on individual factors, which are based on economic models (Wanka \& Gallistl, 2018). Yet, the technology acceptance model (TAM) is widely used and several iterations have been proposed (Legris, Ingham, \& Collerette, 2003; Venkatesh \& Davis, 2000). The initial model (Fig. 1) was later refined to also include "social influence" and amended by factors such as "attitude towards" technology (Legris et al., 2003). It stays close however, to the original assumption of a user as an individual whose preference and usage of a technology can be determined through rational choice. 


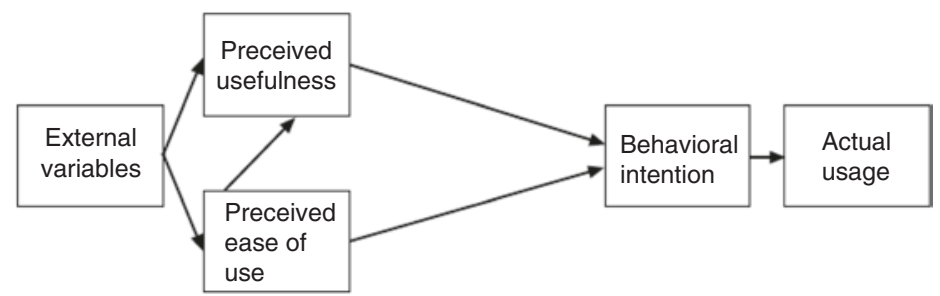

Fig. 1 Original technology acceptance model (Davis \& Venkatesh, 1996)

\section{Technology Use as Embedded in Structural Arrangements}

A second paradigm to understanding old age and technology is based on structuralinstitutional theories, which argue that differences in technology use stem from differences arising from social inequalities (Wanka \& Gallistl, 2018). These inequalities are not individual but are based upon structural arrangements which lead, amongst others, to differences in access to knowledge and a "digital divide" (Friemel, 2014; Neves, Amaro, \& Fonseca, 2012; Zillien \& Hargittai, 2009). In Europe's Digital Progress Report 2017, the European Commission reports that

[i]n 2016, regular internet use grew particularly fast among disadvantaged groups: 63\% of the total in 2015 compared to $60 \%$ a year earlier. 57\% of those aged 55-74 went online at least weekly $[\ldots]$ despite ongoing improvements, the elderly and those with low education levels or on low incomes continue to be at risk of digital exclusion (Human Capital p. 4). ${ }^{1}$

The European Parliament also pointed to the still existing age divide in 2015: From 2005 to 2014 the percentage of internet users among the population has grown for all age groups almost equally and the age gap has remained about $30 \%$. $^{2}$ The full size of this dynamic, however, does not become visible, as the statistics exclude citizens above 74. However, a representative German survey shows that the age of 70 can be considered the turning point where "offliners" become the majority (DIVSI, 2016). While $87 \%$ of those aged 60-64 years have used the internet, only $39 \%$ aged $70-74$ years have, and only $11 \%$ of those older than 80 years were at least occasional internet users.

The European Commission and Member States are aware of an uneven distribution of access and use with regard to gender, age, education and ethnic characteristics as well as regional differences since the late 1990s. The main reason for political action in the field of e-inclusion is the risk of excluding those citizens that are not digitally literate and do not use digital media from the ever-increasing number of digital public services. Based on the assumption that digital exclusion will increase social exclusion several programmes have been set up, to create an "Information Society for all." From the European Council resolution on e-inclusion in October

\footnotetext{
${ }^{1}$ Europe's Digital Progress Report Human Capital Chapter.pdf.

${ }^{2}$ EU Parliament Briefing December 2015: Bridging the Digital Divide in the EU.
} 
2001 and the European Actions Plans to the recent Digital Agenda three areas of action have been highlighted:

- Provide easy and cheap access to the internet from home (broadband) as well as public places,

- Extent and improve digital public/e-government services as incentives,

- Enhance digital skills and literacy for all age groups (lifelong learning).

Overall the factors, listed above are not independent: low awareness of potential benefits from accessing the internet at home prevents people from acquiring the necessary skills and spend money for equipment and access (Kubicek \& Lippa, 2017; Rice \& Katz, 2017). According to the European Digital Progress Report (EDPR):

The three main reasons evoked by households for not having internet access continue to be the lack of need or interest (46\% of households without internet access in 2016), insufficient skills (42\%) and the high costs of equipment (26\%) and access (22\%).

One of the main strategies to overcome the investment dilemma of older adults and to offer the chance of experiencing the benefits of online services have been Public Internet Access Points (PIAPS) at the local level: Libraries, youth and senior centres, community centres and other organisations provide desktop PCs with Internet connection and often some kind of introductory training. According to a survey by Telecentres Europe there were more than 25,000 e-inclusion organisations in the EU 27, one for every 2,000 inhabitants. However, in the age of mobile internet and mobile devices, stationary PIAPS can no longer provide the chance to discover the benefits of this new generation of ICT (Kubicek \& Lippa, 2017).

This paradigm hence provides "life-course perspectives on social inequalities and technology use" (Wanka \& Gallistl, 2018, p. 3). It leads to a construction of old age as a potentially active and autonomous stage, however this potential can only be realised through the appropriate technical solution (Wanka \& Gallistl, 2018). It is in this way, demographic ageing is depicted as promising financial opportunities through the so-called "silver economy" which spurs technical innovation, new products and services (Harper, 2006).

\section{Performing Age Through Technology Design and Use}

A third paradigm relates to post-structural theories (Wanka \& Gallistl, 2018). In this paradigm, researchers move away from "biological and biomedical models of age to re-imagine the complex subjective and culturally mediated ways in which age is embodied, measured, and expressed in multiple and non-chronological ways" (Marshall \& Katz, 2016, p. 146). Within this paradigm, scholars have turned to 
focus on ageing as a social practice - age "is something that people do, not something they are" (Wanka \& Gallistl, 2018, p. 4).

Following Schatzki (2002); Schatzki, Knorr-Cetina, and Von Savigny (2001), the use of technology is understood as a teleoaffective practice-e.g. communicating with family and therefore using a digital messenger. Using a tablet computer does not only rely on the relevant knowledge and skills to use it, but also requires a specific self-image and self-efficacy as a skilled and legitimate user. This self-image or agency is in turn created by actually using a tablet. Low perceived self-efficacy requires targeted actions to enhance digital literacy and have to be much more responsive to individual living circumstances and capabilities (see also Kubicek \& Lippa, 2017).

In a comprehensive review of 644 papers covering human-computer interaction (HCI) and older users, Vines et al. (2015) find that most research and design reinforces particular stereotypes of ageing and subsequently limits "our understanding of how older people might experience, live with, use and actively shape and design technologies both now and in the future" (p. 16).

Most software development projects are based on the designers' assumptions regarding older people's needs. Östlund, Olander, Jonsson, and Frennert (2015) warn that by using such technologies dependencies may be reinforced and older users are kept "hostage". For example, Vines et al. (2015) emphasise the risks associated with reductionist accounts of human beings as users of IT systems:

While defining the user of a new technology can be beneficial in characterising its use cases, it has been long argued that this comes with the danger that heterogeneous and multifaceted human beings are reductively portrayed only in relation to the systems they use and how they are allowed to use them (Vines et al., 2015, p. 2).

Critical scholars in Science and Technology Studies (STS)-inspired social gerontology but also human-computer interaction (HCI) hence demand a more critical engagement with technology design for older adults (e.g. Bischof \& Jarke forthcoming; Neven, 2011; Peine, Faulkner, Jæger, \& Moors, 2015; Vines et al., 2015). In particular, scholars question the representations of "age" that are often scripted into technologies and call attention to the potential consequences of their use. For example, Lucy Suchman (2007) pointed out that information technologies are "sociomaterial configurations" that join together social imaginaries and materialities. Engaging older adults prior to the design process, embraces alternative measures and attributes of "success" in later life and reframes how and which imaginaries are being scripted into technologies (Vines et al., 2015, p. 20; Maaß \& Buchmüller, 2018; Jarke \& Gerhard, 2018).

Thus, the step towards a participatory design perspective is viewed as tremendous progress in the area of technology design for older users. Researchers, developers as well as funding agencies consider the social context of technology development and use as integral part of their agendas. 


\section{Policy Responses in Ageing Societies: Age-Friendly Cities and Communities}

There are a number of policy responses to demographic ageing ranging from later retirement age and lifelong learning to approaches on local level covering agefriendly cities and communities. According to the World Health Organization (WHO), a more supportive and enabling social and physical environment is essential for people to age in better conditions. The WHO Age-friendly Cities and Communities approach proposes a framework of eight interconnected domains that shall help to identify and address barriers to the well-being and participation of older people: built environment and outdoor spaces; housing; transportation; social participation; respect and social inclusion; civic participation and employment; communication and information; and community support and health services (Fig. 2).

By adapting environments to the WHO approach, the following objectives are pursued:

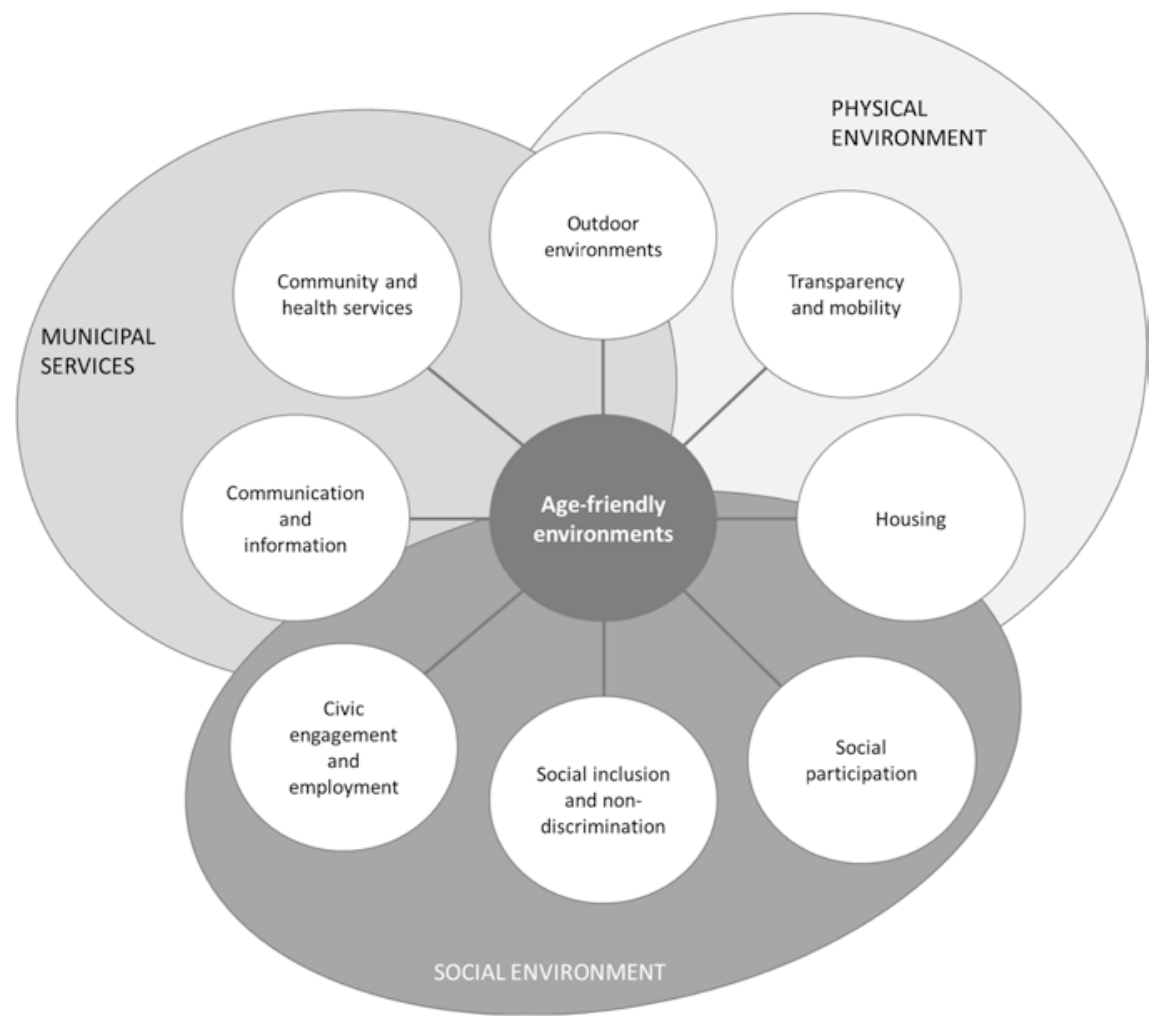

Fig. 2 WHO age-friendly cities guidelines (adapted from WHO, 2017) 
- Enable older workers to remain at work for longer even those facing chronic diseases, reduced mobility or other limitations in their daily activities; enable workers who care for older relatives (often women aged 50+) to remain at work for longer, protect their own health and build a decent pension for their own old age.

- Empower older adults to age in better physical and mental health, promote their social inclusion and active participation and help them maintain their autonomy and a good quality of life in their old age even when they become frail(er) and suffer from chronic and age-related diseases.

- Lower the pressure on traditional care and assistance services, and support longer independent living.

- Create new jobs and growth opportunities through demand for innovative solutions in the silver economy sectors such as health and long-term care, housing, transport, culture, tourism, IT.

Technology, however, has not been sufficiently addressed in the WHO model. For example, the fields "social inclusion" and "civic participation and employment" do not include "essential features related to technology" (Marston \& van Hoof, 2019, p. 7). This book demonstrates that questions around social inclusion as well as social participation are inherently also questions of digital inclusion and digital participation. Hence, participation in the design of technological futures is important for ensuring social participation. One policy field in which this becomes increasingly important are smart cities. A number of civil society organisations such as the AGE Platform Europe, the EU Covenant on Demographic Change, and organisations such as the WHO Global Network of Age-Friendly Cities and Communities (WHO GNAFCC) already promote a more inclusive approach to smart city developments. Co-creation may be one way to follow-up on the promise of engaging older citizens in inclusive future-making.

Open Access This chapter is licensed under the terms of the Creative Commons Attribution 4.0 International License (http://creativecommons.org/licenses/by/4.0/), which permits use, sharing, adaptation, distribution and reproduction in any medium or format, as long as you give appropriate credit to the original author(s) and the source, provide a link to the Creative Commons license and indicate if changes were made.

The images or other third party material in this chapter are included in the chapter's Creative Commons license, unless indicated otherwise in a credit line to the material. If material is not included in the chapter's Creative Commons license and your intended use is not permitted by statutory regulation or exceeds the permitted use, you will need to obtain permission directly from the copyright holder. 